\title{
Various sizes of sliding event bursts in the plastic flow of metallic glasses based on a spatiotemporal dynamic model
}

\author{
Jingli Ren, ${ }^{1, a)}$ Cun Chen, ${ }^{1}$ Gang Wang, ${ }^{2, a)}$ Wing-Sum Cheung, ${ }^{3}$ Baoan Sun, ${ }^{4}$ \\ Norbert Mattern, ${ }^{4}$ Stefan Siegmund, ${ }^{5}$ and Jürgen Eckert ${ }^{4,6}$ \\ ${ }^{1}$ School of Mathematics and Statistics, Zhengzhou University, Zhengzhou 450001, China \\ ${ }^{2}$ Laboratory for Microstructures, Shanghai University, Shanghai 200444, China \\ ${ }^{3}$ Department of Mathematics, The University of HongKong, HongKong, China \\ ${ }^{4} I F W$-dresden, Institute for Complex Materials, P.O. Box 2701 16, D-01171 Dresden, Germany \\ ${ }^{5}$ Department of Mathematics, TU Dresden, D-01062 Dresden, Germany \\ ${ }^{6}$ Institute of Materials Science, TU Dresden, D-01062 Dresden, Germany
}

(Received 11 June 2014; accepted 9 July 2014; published online 21 July 2014)

\begin{abstract}
This paper presents a spatiotemporal dynamic model based on the interaction between multiple shear bands in the plastic flow of metallic glasses during compressive deformation. Various sizes of sliding events burst in the plastic deformation as the generation of different scales of shear branches occurred; microscopic creep events and delocalized sliding events were analyzed based on the established model. This paper discusses the spatially uniform solutions and traveling wave solution. The phase space of the spatially uniform system applied in this study reflected the chaotic state of the system at a lower strain rate. Moreover, numerical simulation showed that the microscopic creep events were manifested at a lower strain rate, whereas the delocalized sliding events were manifested at a higher strain rate. (C) 2014 AIP Publishing LLC.
\end{abstract}

[http://dx.doi.org/10.1063/1.4890720]

\section{THEORETICAL BACKGROUND}

The plastic deformation of metallic glasses is typically manifested as a series of intermittent serrations on a stressstrain (time) curve, which is induced by the operation of shear bands. ${ }^{1}$ This serrated deformation is composed of an accumulation of elastic energy and an energy relaxation. ${ }^{2}$ Statistical analysis of the time-series of serrated stress-time curves, which were obtained by studying metallic glasses with various levels of ductility, showed that the dynamic behavior in the brittle sample was in a chaotic state, and that the plasticity could be attributed to the stick-slip of a single shear band. Regarding the ductile sample, the distributions of stress drops and their time durations conformed to a power-law scaling, suggesting a self-organization critical (SOC) state, and the plasticity was attributed to the simultaneous nucleation of numerous shear bands and their mutual interactions. ${ }^{3-5}$ Further analysis of the stress-time series regarding the serrated flow of metallic glasses revealed that the dynamics of serrated flow in metallic glasses behaved in such a manner that there was a transition from a chaotic state to a SOC state via a multifractal intermediate when strain rates were increasing or temperatures were decreasing. ${ }^{6}$

The operation of serrated deformation is believed to be associated with some operatively primary deformation units embedding in the glassy phase. In order to quantitatively describe the deformation mechanism of metallic glasses, a number of theories such as the shear transformation zone (STZ) theory, ${ }^{7}$ the flow units, ${ }^{8}$ the free volume theory have been proposed. ${ }^{9}$ After analysing the elastic energy aggregation

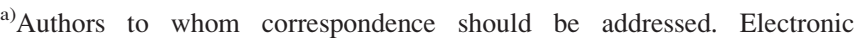
addresses: renjl@zzu.edu.cn and g.wang@shu.edu.cn
}

and release in serration events, a phenomenological model was also established to schematically examine the correlation between the shear banding and serration events of metallic glasses. ${ }^{10}$ Recently, through investigation of the anelastic relaxation of metallic glasses, Atzmon et al. revealed a quantized hierarchy of STZs differ from each other by one atomic volume. ${ }^{11}$ Moreover, based on the analysis of the homogenous deformation of metallic glasses at room temperature, a flow unit model was proposed by Lu et al. ${ }^{8}$ Although the potential energy landscape (PEL) theory have been proposed to correlate the primary deformation unit with the macroscopic shear deformation, ${ }^{12}$ a phenomenological model is significantly needed to describe the dynamics of the stochastically intermittent serrated flow in metallic glasses.

In 1967, Burridge and Knopoff originally introduced a model to explore the role of friction along a fault as a factor in the earthquake mechanism; ${ }^{13}$ using the prescribed model, ${ }^{13}$ Carlson and Langer presented a deeper understanding of behavior for an earthquake fault, which consists of an elastically coupled chain of masses in contact with a moving rough surface. ${ }^{14,15}$ For the metal glasses, Cheng et al. got a model by discussing the increase in temperature in the major shear band; ${ }^{10}$ Sun et al. proposed a dynamic model for ductile metallic glasses by considering the interaction of multiple shear bands, ${ }^{16}$ and deduced a simple expression for the mutual interaction between the shear bands in a tick-slip model. ${ }^{17}$ Besides, Antonaglia et al. showed that bulk metallic glasses deform via slip avalanches by demonstrating agreement of high temporal resolution measurements of the slip-statistics and dynamics with the predictions of a simple mean field model for plastic deformation. ${ }^{18}$

The aim of the present work is to establish a dynamic model based on the interaction between multiple shear 
bands, as well as the related loading strain rate effects. The model focuses on the heterogeneous deformation of metallic glasses at room temperature that is far lower than the glassy transition temperature, $T_{\mathrm{g}}$, (i.e., the deformation temperature is lower than $0.8 T_{\mathrm{g}}$ ). The novel aspect of this work relates to two factors: (1) a qualitative analysis was conducted to evaluate the instability of the solution under varying conditions and to explain the unstable chaotic mechanism based on dynamic analysis; (2) various sizes of sliding events can be deduced from this model at different strain rates.

Considering the mutual interaction between multiple shear bands, a model of shear banding was created, which involved representing the shear displacement and the time as a sketch, as shown in Fig. 1. This sketch depicts a chain of blocks that were coupled to each other by harmonic springs with strength of $k_{c}$ and were attached to the machine. The spring strength between the sample and the machine is $k$ (Figs. 1(a) and 1(b)). The system is compressed at a loading speed, $v$, as shown in Fig. 1(a). For the $i$ th block, the forces parallel to the loading direction are the internal stress, $\sigma\left(U_{i}\right)$ (where $U_{i}$ is the shear sliding displacement of the $i$ th block), the interaction among the multiple shear bands, which can be expressed as $U_{i+1}+U_{i-1}-2 U_{i}$, and the plastic shear resistance, $\sigma_{f}\left(\dot{U}_{i}\right)$, which is in the opposite direction of the loading direction (Fig. 1(c)). Because the internal stress, $\sigma\left(U_{i}\right)$, can be written as $\sigma\left(U_{i}\right)=\sigma(0)-k U_{i},{ }^{10}$ the motion equation is as follows:

$$
\frac{4 M}{\pi d^{2}} \ddot{U}_{i}=\sigma(0)-k U_{i}+k_{c}\left(U_{i+1}+U_{i-1}-2 U_{i}\right)-\sigma_{f}(\dot{U}),
$$

where $\sigma(0)$ is the initial internal stress, which is equal to the yield stress, $\sigma_{f 0}$, and $\sigma_{f}(\dot{U})=\frac{\sigma_{f 0}}{1+A \dot{U}}$, which represents the shear resistance along the shear plane. ${ }^{16} d$ is the diameter of the sample. $M$ is the equivalent mass of the system, which consists of a metallic glass and a spring that represents the influence of the testing machine. $k=E / L(1+S)$ (here, $E$ is the Young modulus of the metallic glass, and $S$ is the stiffness ratio of sample $\kappa_{\mathrm{S}}$ to the testing machine $\kappa_{\mathrm{M}}$; i.e., $\left.S=\kappa_{\mathrm{S}} / \kappa_{\mathrm{M}}=\pi d^{2} / E / 4 L \kappa_{\mathrm{M}}\right){ }^{19}$

On a macro-scale, metallic glasses behave in the manner of an isotropic continuum solid, ${ }^{20}$ which facilitates evaluating the glassy phase in the framework of a continuous system. In the current study, a differential equation describing (a)

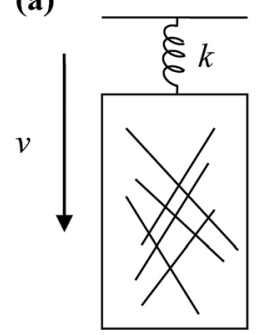

(b)

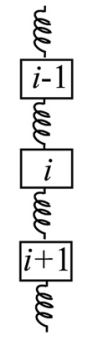

(c)

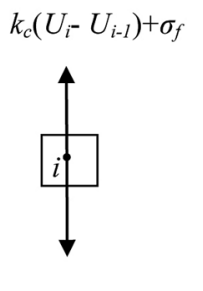

$k_{c}\left(U_{i+1^{-}} U_{i}\right)+\sigma$
FIG. 1. The schematic diagram of the plastic deformation and stress analysis in the multiple shear bands. (a) The multiple shear bands during the plastic deformation. (b) Chain of the blocks parallel to loading direction. (c) The force diagram for the $i$ th block. the kinetics of shear banding was constructed. The expression $x=i h$ was adopted as a dimensionless variable representing the position of the $i$ th block, where $h$ is the length between each block. It can be deduced using Eq. (1)

$$
\frac{4 M}{\pi d^{2}} U_{t t}=\sigma(0)-k U+k_{c} h^{2} U_{x x}-\sigma_{f}\left(U_{t}\right) .
$$

The remainder of this paper is described as follows: Spatially uniform solutions and traveling wave solutions are discussed in Secs. II and III. In Sec. IV, these sliding events are categorized as being small, (i.e., microscopic creeping events) and large (i.e., delocalized sliding events), and the two types of sliding events are discussed. Section V presents the results of analyzing a numerical simulation, suggesting that various sizes of events correspond to the microscopic creeping events and delocalized sliding events discussed in Sec. IV.

\section{SPATIALLY UNIFORM SOLUTION}

A solution $U(x, t)$ is spatially uniform if it is constant in the position variable $x$, which implies $U_{x x}=0$. A spatially uniform solution $U(t)$ of Eq. (2) satisfies

$$
\frac{4 M}{\pi d^{2}} \ddot{U}(t)=\sigma(0)-k U-\frac{\sigma_{f 0}}{1+A \dot{U}} .
$$

A numerical solution of this equation is plotted in Fig. 2(a), which shows the displacement, $U$, as a function of $t$ at $v=10^{-7} \mathrm{~m} / \mathrm{s}$ (where $v$ is the loading speed). The sliding velocity suggests that the system alternately sticks and slips in unison, as if it were a single block, as shown in Fig. 2(b). Based on stability analysis, this uniform stick-slip motion is unstable. ${ }^{21}$

Figure 3(a) shows a plot of the phase diagram of the system (3) at $t \in[0,0.3]$ and $v=10^{-7} \mathrm{~m} / \mathrm{s}$. The cycles in the phase plane were determined to be limited, suggesting that the system (3) exhibited periodic solutions and a chaotic attractor. The characteristics of the attractor are explained as follows. At a high sliding speed, such as $v=100 \mathrm{~m} / \mathrm{s}$, the phase diagram of the system indicated that the system contained several limit cycles, as shown in Fig. 3(b), resulting in the periodic solutions for the system.

The strain-time curve of the $\mathrm{Cu}_{50} \mathrm{Zr}_{45} \mathrm{Ti}_{5}$ metallic glass, which demonstrated a strain rate of $2.5 \times 10^{-5} \mathrm{~s}^{-1}$, is represented in Fig. 4(a). The plot of $(\dot{\varepsilon}, \varepsilon)$ (where $\varepsilon$ and $\dot{\varepsilon}$ are the strain and strain rate, respectively) is shown in Fig. 4(b), which displays an attractor that is similar in appearance to the phase diagram in Fig. 3(a). Therefore, the phase space of the system (3) exhibited a similar to that of the experiment, suggesting that the uniform solution reasonably reflected the characteristics of the original system (2).

\section{TRAVELING WAVE SOLUTION}

The propagation of a shear band generates shear offset traveling across the sample, which is too fast to be observed directly. ${ }^{22}$ In the current study, the shear band was considered a wave propagating in the sample. The interactions between shear bands resemble the transmission of waves in a 

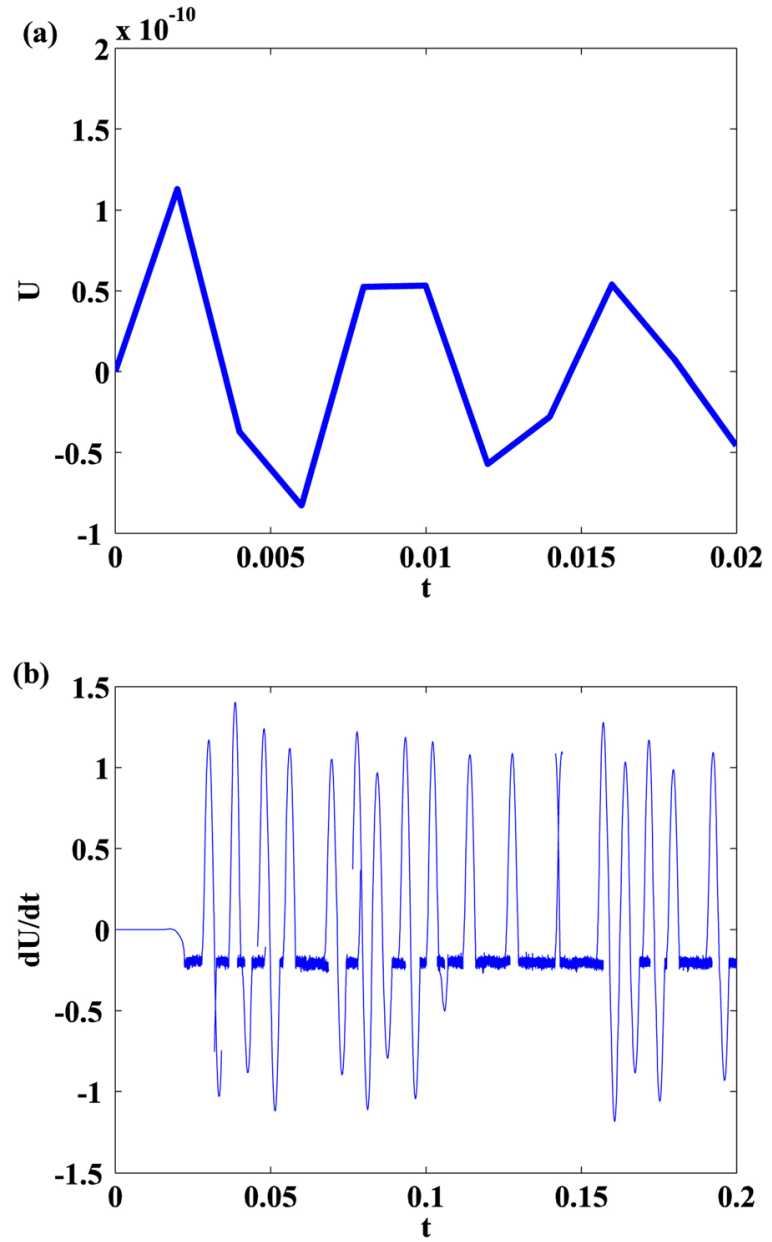

FIG. 2. (a) The spatially uniform solution of system (2) is plotted in which the displacement $U$ is a function of $t$ for $v=10^{-7} \mathrm{~m} / \mathrm{s}$. (b) The velocity $\mathrm{d} U /$ $\mathrm{d} t$ as a function of $t$ for $v=10^{-7} \mathrm{~m} / \mathrm{s}$.

homogeneous isotropic elastic body. ${ }^{23}$ Thus, to solve the partial differential Eq. (2), the strain evolution was traced during the deformation by analyzing the traveling wave transformation.

According to the traveling wave transformation, $\xi=x-c t$, where $c$ is the speed of the traveling wave, $U(x, t)=U(\xi)$. Equation (2) can be translated into the following ordinary differential equation, which enables obtaining solutions in the form of propagating kinks

$$
\left(k_{c} h^{2}-\frac{4 M c^{2}}{\pi d^{2}}\right) U^{\prime \prime}-k U-\frac{\sigma_{f 0}}{1-A c U^{\prime}}+\sigma(0)=0 .
$$

When the slipping velocity is adequately low, the linearization of Eq. (4) at the uniform solution of $U=\left(\sigma(0)-\sigma_{f 0}\right) / k$ is

$$
\left(k_{c} h^{2}-\frac{4 M c^{2}}{\pi d^{2}}\right) U^{\prime \prime}-\sigma_{f 0} A c U^{\prime}-k U=0 .
$$

Considering $k_{c} h^{2}>4 M c^{2} / \pi d^{2}$, the solution of Eq. (5) is

$$
U(\xi)=c_{1} \exp \left(\lambda_{1} \xi\right)+c_{1} \exp \left(\lambda_{2} \xi\right)
$$

where $\lambda_{1,2}=\left(A c \sigma_{f 0} \pm \sqrt{\left.\left(A c \sigma_{f 0}\right)^{2}+4 k\left(k_{c} h^{2}-4 M c^{2} / \pi d^{2}\right)\right)} /\right.$ $\left(2\left(k_{c} h^{2}-4 M c^{2} / \pi d^{2}\right.\right.$ and $c_{1}, c_{2}$ are constant.
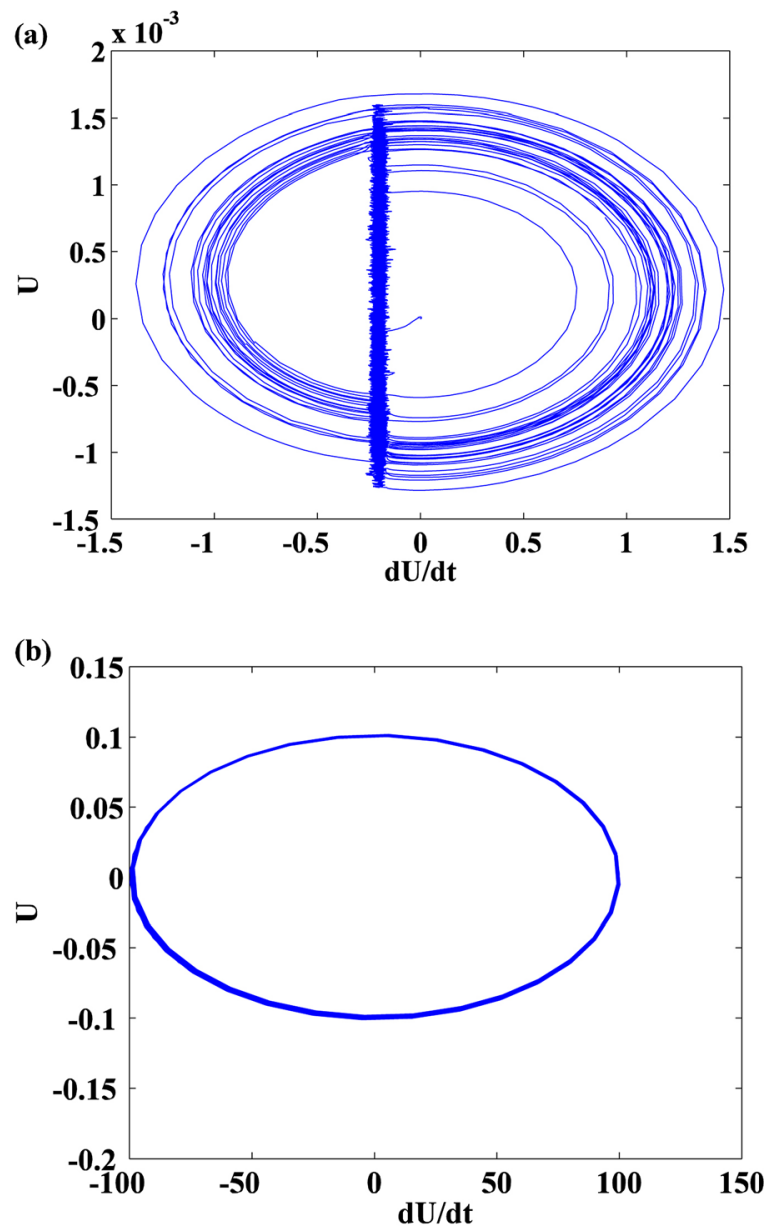

FIG. 3. (a) The phase diagram of system (3) at low sliding speed $v=10^{-7}$ $\mathrm{m} / \mathrm{s}$. (b) The phase diagram of the system (3) at large sliding speed $v=100 \mathrm{~m} / \mathrm{s}$.

When $k_{c}$ is larger than $4 M c^{2} /\left(\pi d^{2} h^{2}\right)$, the displacement of the shear bands grows exponentially fast. These traveling wave solutions remain in an unstable state, similar to the aforementioned spatial uniform solutions. Any small irregularities in the positions of the blocks, regardless of the wavelength, are amplified while the system is thus slipping.

\section{MICROSCOPIC CREEPING EVENTS AND DELOCALIZED SLIDING EVENTS}

In a real system, the slipping events that occur in compressed metallic glass are not always spatially uniform or in the form of propagating kinks. These events can be described as localized, and the delocalized process is on a micro-scale. Examining these events analytically is necessary, in addition to performing numerical simulations. These microscopic creeping events involve only one or two blocks, and the sliding speed is relatively low. In the current study, the microscopic creeping events were represented in system (1) in which such events were visible.

When a connected group includes $n$ blocks that move uniformly, this group can slip as integrity. Such a slipping event occurs when the group has fallen far away from its neighbors. The combined forces exerted by the coupling springs are equal to the shear resistance.

The position of the mass center of this group, $U_{c . m}$, can be expressed as 

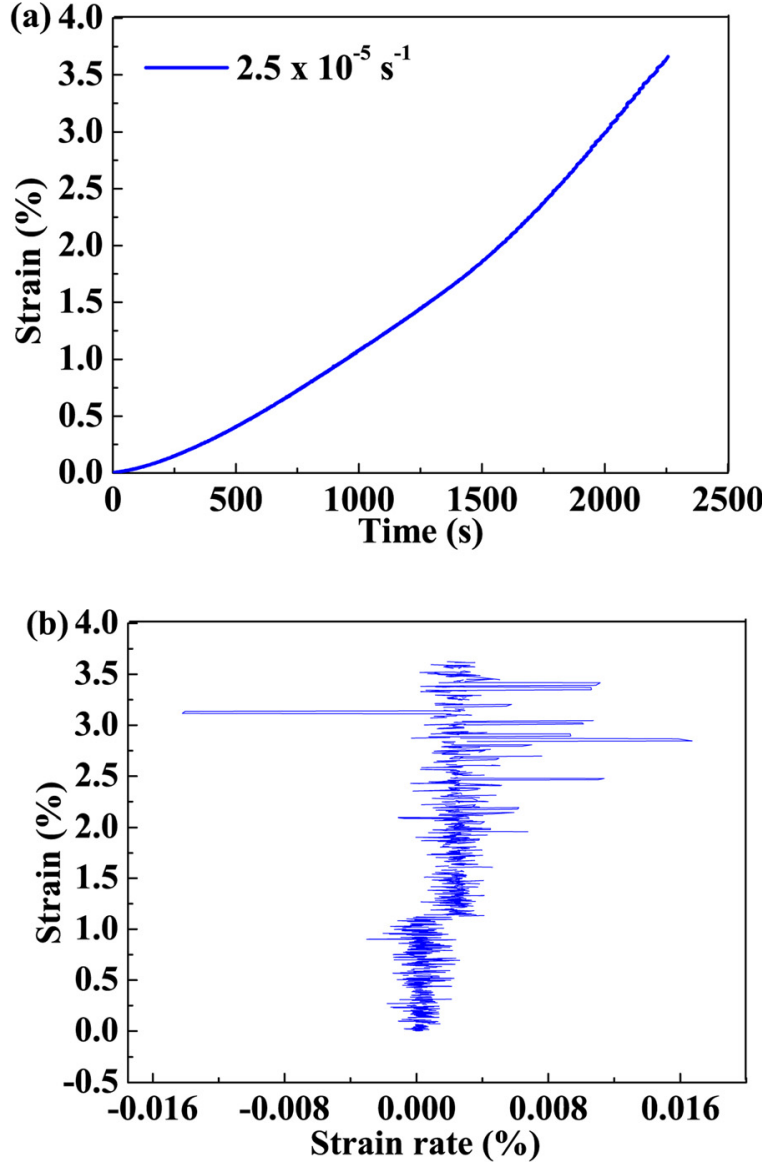

FIG. 4. (a) The strain-time curve of the $\mathrm{Cu}_{50} \mathrm{Zr}_{45} \mathrm{Ti}_{5}$ metallic glass strained at the strain rate of $2.5 \times 10^{-5} \mathrm{~s}^{-1}$. (b) Plot of the strain vs. the strain rate.

$$
U_{c . m .}=\frac{1}{n} \sum_{j=j_{1}}^{j_{1}+n} x_{j}=-v t+\dot{W}_{n} .
$$

Note that we shifted the frame of reference in which $\dot{W}_{n}=0$ when the blocks are stuck. The equation of motion for $W_{n}$ is

$$
\frac{4 M}{\pi d^{2}} \ddot{W}_{n}-\sigma_{f 0} A \dot{W}_{n}+\left(k+\frac{2 k_{c}}{n}\right) W_{n}-k v t+\sigma_{f 0} A k v=0 .
$$

The origin of the time axis is used as the point at which the system can start slipping, with $W_{n}(0)=\dot{W}_{n}(0)=0$. The form of the equation can be understood by noting that the pulling force of the springs and the shear resistance are applied equally on all of the slipping blocks. For any $n$ block, only the two coupling springs that connect the slipping blocks with the rest of the system can influence the center of mass. Thus, in Eq. (7), the relative effect of the coupling springs, $\frac{2 k_{c}}{n}$, decreases as $n$ increases. The solution of Eq. (7) is as follows:

$$
\begin{aligned}
W_{n}(t)= & c_{1} \frac{\alpha e^{\alpha t} \cos \beta t+\beta e^{\alpha t} \sin \beta t}{\alpha^{2}+\beta^{2}} \\
& +c_{2} \frac{\alpha e^{\alpha t} \sin \beta t-\beta e^{\alpha t} \cos \beta t}{\alpha^{2}+\beta^{2}}+\frac{k v t-\sigma_{f 0} A k v}{k+2 k_{c} / n} \\
& +\frac{\sigma_{f 0} A k v}{\left(k+2 k_{c} / n\right)^{2}}
\end{aligned}
$$

where $\alpha=\operatorname{Re}(\lambda), \beta=\operatorname{Im}(\lambda)$,

$$
\begin{gathered}
\lambda=\left(\sigma_{f 0} A \pm \sqrt{\left.\left(\sigma_{f 0} A\right)^{2}-16 M\left(k+2 K_{c} / n\right) /\left(\pi d^{2}\right)\right)} /\left(8 M /\left(\pi d^{2}\right)\right),\right. \\
c_{1}=\frac{-k v}{k+2 k_{c} / n}, \\
c_{2}=\frac{\alpha^{2}+\beta^{2}}{\beta\left(k+2 k_{c} / n\right)}\left(\frac{\sigma_{f 0} A k v}{k+2 k_{c} / n}-\frac{\alpha k v}{\alpha^{2}+\beta^{2}}-\sigma_{f 0} A k v\right) .
\end{gathered}
$$

It can be deduced that $\dot{W}_{n}(t)=c_{1} e^{\alpha t} \cos \beta t$ $+c_{2} e^{\alpha t} \sin \beta t+k v /\left(k+2 k_{c} / n\right)$, and $\dot{W}_{n}(t)<v$ for small $n$. Although a group of blocks undergoing a sequence of these small periodic events catches up with its neighbors, it falls increasingly further behind the average displacement of the system.

The assumption that for large $n$ values the slipping blocks can be spaced uniformly is unrealistic. For small $n$ values, the strain and the energy stored in the coupling springs were assumed to be strongly localized at the ends of the slipping region. To obtain the analytic result of this dynamic process, we refer to Eq. (2). According to Eq. (2), the condition of the blocks being on the edge of slipping is expressed as

$$
k_{c} h^{2} \frac{d^{2} U}{d x^{2}}-k U=0
$$

meaning that the sum of the coupling and pulling forces balances the maximum static friction continuously across the entire slipping zone. The solution of Eq. (9) can be written as

$$
U_{\varepsilon}(x)=\varepsilon \cosh \left(\sqrt{k /\left(k_{c} h^{2}\right)} x\right),
$$

where $\varepsilon$ is a constant.

A slipping zone is used in a local region where Eq. (9) can be satisfied. The center of this slipping zone is at $x=0$. Within this zone, where a slipping event nearly occurs, $U(x, t)=U_{\varepsilon}(x)+u(x, t)$ can be written. Solving Eq. (2) yields the following linearized system:

$$
\frac{4 M}{\pi d^{2}} u_{t t}-k_{c} h^{2} u_{x x}-\sigma_{f 0} A u_{t}+k u=0 .
$$

$U_{\varepsilon}(x)$ is the edge of the slipping zone; therefore, the slipping motion is triggered by a low pulse $u(x, t)$ at a certain position, which we assumed to be $x_{0}$. In a real system, this slipping motion may be triggered by many low, simultaneous pulses. Based on linear approximation, such a motion is regarded as a linear superposition of the motions generated by single pulses, which is consistent with the fact that the interaction between the shear bands can be treated as the superposition of two elastic strain fields. ${ }^{24,25}$ For simplicity, we describe this event according to the initial conditions of $u(x, 0)=0, \dot{u}(x, 0)=w_{0} \delta\left(x-x_{0}\right)$, where $w_{0}$ is proportional to the slipping speed of the triggering event. The subsequent motion is expressed as

$$
u(x, t)=\frac{w_{0}}{2 \pi} \int_{-\infty}^{+\infty} \frac{\sin \Gamma_{q} t}{\Gamma_{q}} e^{i q\left(x-x_{0}\right)+\alpha t} d q,
$$


where $q$ is a wave number, $\alpha=\pi d^{2} A \sigma_{f o} /(8 M)$,

$$
\Gamma_{q}=\frac{1}{2} \sqrt{\pi d^{2}\left(k+k_{c} h_{x}^{2} q^{2}\right) / M-\left(\pi d^{2} \sigma_{f 0} A /(4 M)\right)^{2}} .
$$

Using Eq. (12) yields

$$
\dot{u}(x, t)=\frac{w_{0}}{2 \pi} \int_{-\infty}^{+\infty}\left(\cos \Gamma_{q} t+\frac{\sin \Gamma_{q} t}{\Gamma_{q}} \alpha\right) e^{i q\left(x-x_{0}\right)+\alpha t} d q,
$$

when $\pi d^{2} k_{c} h^{2} q^{2} / M \gg \pi d^{2} k+\left(\pi d^{2} \sigma_{f 0} A / 4 M\right)^{2}, \Gamma_{q}$ can be approximated as a linear function of $q$

$$
\begin{aligned}
\dot{u}(x, t) \approx & \frac{w_{0}}{2} e^{\alpha t}\left[\delta\left(x-x_{0}-\sqrt{\pi d^{2} k_{c} h_{x}^{2} / 4 M t}\right)\right. \\
& \left.+\delta\left(x-x_{0}+\sqrt{\pi d^{2} k_{c} h_{x}^{2} / 4 M t}\right)\right] .
\end{aligned}
$$

When the slipping zone is small, the pulses must stop at the edges of the sample. The pulses cannot increase adequately to drive the neighboring stuck blocks to a point of slipping, and the slipping remains localized. Moreover, low propagating pulses must be extremely noisy because of the instability caused by the nonlinear term of $\sigma_{f}(\dot{U})$.

However, in an extremely large zone, where $\varepsilon$ is excessively small so that $U$ is close to its limiting value of 0 across a large part of the system, a pulse may become delocalized and push the blocks outside the initial slipping zone. When the sliding speed becomes adequately high, the linear approximation in Eq. (7) fails. The amplification of the pulse is then weakened and the solution is similar to the propagating solutions of Eq. (4). We observed localized and delocalized events in the numerical simulations.

A qualitative description of how chaotic behavior is generated in this model is provided as follows. A shear band, with a scale of $\Delta x$, amplifies the irregularities on the scales that are smaller than $\Delta x$. These irregularities subsequently produce more of the smaller events. Simultaneously, because the blocks that slip in the zone of $\Delta x$ generally catch up with their neighbors, the event can smoothen the system on scales larger than $\Delta x$ and accumulate into a larger scale event. Thus, events of various sizes are generated irregularly and persistently by the deterministic motion of this dynamic system. To explain this complex behavior further, Sec. V discusses the numerical methods used in this study.

\section{NUMERICAL VERIFICATION}

The discrete Eq. (1) is solved numerically for various choices of the parameters under the periodic boundary conditions of $U(x, 0)=0, U(0, t)=U(L, t)$, and $U_{t}(x, 0)=v$.

Generally, events with various sizes are observed, and some of them are shown in Figs. 5(a)-5(c) in the form of the sliding velocity of $\dot{U}$ as a function of positions $i$ and time $t$. The parameters used in the numerical simulations were $v=10^{-7} \mathrm{~m} / \mathrm{s} \sim 10^{-4} \mathrm{~m} / \mathrm{s}$, and $N=100$.

For the lower strain rate of $2.5 \times 10^{-5} \mathrm{~s}^{-1}$, the initial speed is $v=10^{-7} \mathrm{~m} / \mathrm{s}$. Figure 5 (a) demonstrates the microscopic creeping events with only one or two blocks sliding fast. Because the vertical scale was greatly expanded, the maximum sliding velocity of all the events was less than the pulling speed $v$. The most distinctive feature in Fig. 5(a) is two larger events involving more than one block. Microscopic creeping events are corresponding to that described by Eq. (8). The events of more than one block are rare.

At the middle strain rate of $2.5 \times 10^{-3} \mathrm{~s}^{-1}$ and the loading speed of $v=10^{-5} \mathrm{~m} / \mathrm{s}$, a moderately large localized event occurred, as shown in Fig. 5(b). The maximum speed was much higher than that shown in Fig. 5(a), and the one-block and two-block events were much less.
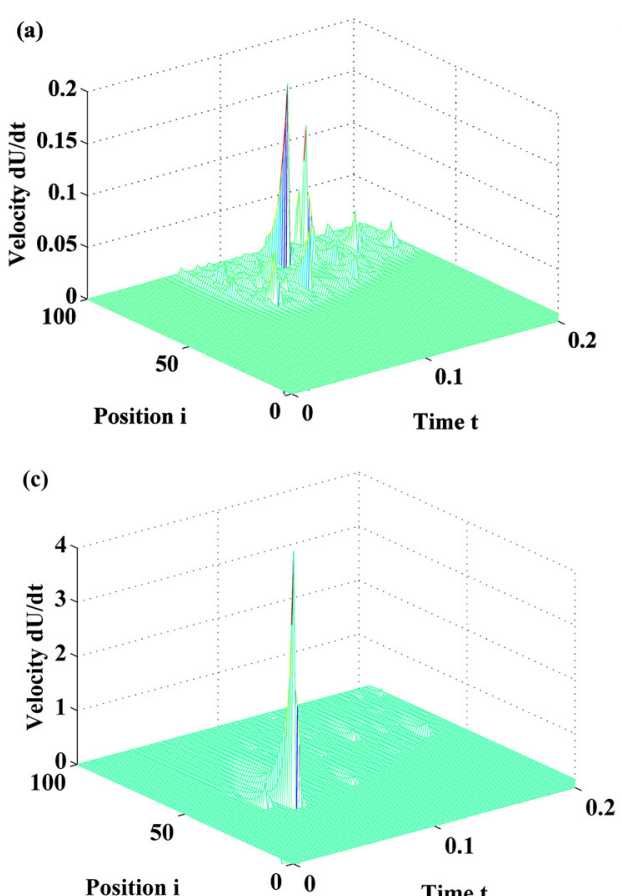

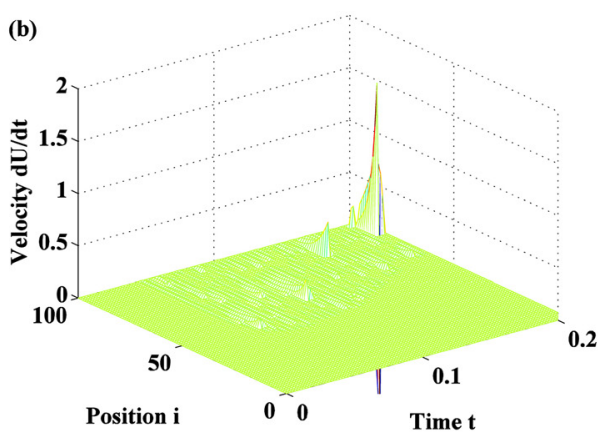

\footnotetext{
FIG. 5. Various sizes of events at strain rates of (a) $2.5 \times 10^{-5} \mathrm{~s}^{-1}$, (b) $2.5 \times 10^{-3} \mathrm{~s}^{-1}$ and (c) $2.5 \times 10^{-2} \mathrm{~s}^{-1}$, $N=100$. The numerical values of the materials parameters used here are $d=2 \mathrm{~mm}, L=4 \mathrm{~mm}, \sigma_{f 0}=1530 \mathrm{MPa}$, $E=80 \mathrm{GPa}, M=8.29 \times 10^{-5} \mathrm{~kg}$.
} 


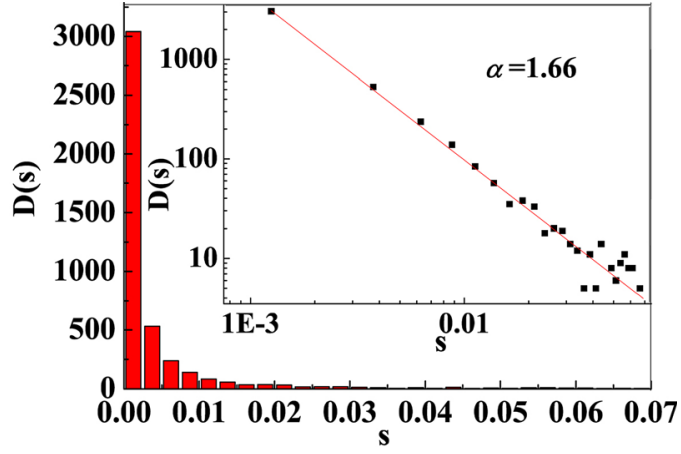

FIG. 6. The distribution $(D(s))$ vs. the sliding speed of each block at the strain rate of $2.5 \times 10^{-2} \mathrm{~s}^{-1}$, where $D(s)$ represents the number of blocks with sliding speed, $s$.

For the higher strain rate of $2.5 \times 10^{-2} \mathrm{~s}^{-1}$ and the loading speed of $v=10^{-4} \mathrm{~m} / \mathrm{s}$, a large delocalized event occurred, as shown in Fig. 5(c). This particular event is reasonably described using Eq. (14), which was triggered by a small event in the interior of the system and then grew exponentially to be a pulse as it propagated in both directions toward the edges. At this point, the maximum slipping speed approached a high value. The event finally vanished as the moving pulses were reflected from the boundaries.

Moreover, based on the numerical results, the statistics of the sliding speed of the $i$ th blocks $\dot{u}_{i}$ (here $\dot{u}_{i}$ is denoted by $s$ for convenience) show a power-law distribution with the fitting exponent $\alpha=1.66$ (Fig. 6). Using $\Delta \sigma=k(s-v) \Delta t$ enables deducing that the stress drops showed a power-law distribution (in other words, the SOC state) at the higher strain rate of $2.5 \times 10^{-2} \mathrm{~s}^{-1}$.

The spatiotemporal dynamic model based on the interaction between multiple shear bands discussed above does not include the influences of the microstructures of glassy phase. Actually, it is generally accepted that the glassy phase behaves dynamic heterogeneities at the atomic scale, ${ }^{26,27}$ which is induced by some heterogeneously primary deformation units. ${ }^{28}$ Considering these heterogeneous units, we introduce the springs with different elastic coefficients, $k_{\mathrm{i}}, i=1$, $2, \ldots, \mathrm{N}-1$, connecting the neighboring blocks into the model, which is,

$$
\begin{aligned}
\frac{4 M}{\pi d^{2}} \ddot{U}_{i}= & \sigma(0)-k U_{i}+k_{i}\left(U_{i+1}-U_{i}\right) \\
& -k_{i-1}\left(U_{i}-U_{i-1}\right)-\sigma_{f}\left(\dot{U}_{i}\right) .
\end{aligned}
$$

Due to the limitation of the experimental method, so far the value of elastic coefficients of $k_{\mathrm{i}}$ is hard to identify, and Eq. (15) is difficult to be transformed into a partially differential equation. Therefore, the currently theoretical analysis will be unable to deal with the model described by Eq. (15). Further theoretical analysis is required to introduce the inhomogeneous structures of the glassy phase into the dynamic model in future studies.

\section{CONCLUSION}

This paper presents a dynamic model that revealed the mechanism causing the occurrence of various sizes of events and the instability of the plastic dynamics in metallic glasses. Spatially uniform solutions and traveling wave solutions are deduced for determining the features of stick-slip behavior and propagating kinks in plastic deformation; in particular, the phase diagram of the spatially uniform system indicates a chaotic state at a lower strain rate. For the microscopic events that evolve a lower number of blocks, the sliding speed is lower than $v$, whereas the delocalized events with higher sliding speeds manifest as the superposition of a low amplitude and exponential pulse. The dynamic analysis of different sizes of events provides a clear mathematical picture to describe the motion of the shear banding behavior in BMGs. Moreover, various sizes of events at different sliding speeds can be observed based on the numerical simulation of the model, and statistically analyzing the numerical results verifies the SOC state at the higher strain rate.

\section{ACKNOWLEDGMENTS}

The work described in this paper is supported by the NSFC (Grants Nos. 11271339, 51171098, and 51222102), the NCET (10-0141) program and the ZDGD13001 program. J. L. Ren, B. A. Sun, and G. Wang acknowledge the Alexander von Humboldt Foundation. The authors thank H.-J. Klauß, S. Donath, M. Frey, and B. Opitz for technical assistance.

${ }^{1}$ D. Klaumünzer, A. Lazarev, R. Maaß, F. H. Dalla Torre, A. Vinogradov, and J. F. Löffler, Phys. Rev. Lett. 107, 185502 (2011).

${ }^{2}$ J. J. Lewandowski and A. L. Greer, Nature Mater. 5, 15 (2006).

${ }^{3}$ R. Sarmah, G. Ananthakrishna, B. A. Sun, and W. H. Wang, Acta Mater. 59, 4482 (2011).

${ }^{4}$ G. Wang, K. C. Chan, L. Xia, P. Yu, J. Shen, and W. H. Wang, Acta Mater. 57, 6146 (2009).

${ }^{5}$ J. L. Ren, C. Chen, G. Wang, N. Mattern, and J. Eckert, AIP Adv. 1, 032158 (2011).

${ }^{6}$ J. L. Ren, C. Chen, Z. Y. Liu, R. Li, and G. Wang, Phys. Rev. B 86, 134303 (2012).

${ }^{7}$ C. A. Schuh and A. C. Lund, Nature Mater. 2, 449 (2003).

${ }^{8}$ Z. Lu, W. Jiao, W. H. Wang, and H. Y. Bai, "Flow unit perspective on room temperature homogeneous plastic deformation in metallic glasses," Phys. Rev. Lett. (to be published).

${ }^{9}$ F. Spaepen, Acta Metall. 25, 407 (1977).

${ }^{10}$ Y. Q. Cheng, Z. Han, Y. Li, and E. Ma, Phys. Rev. B 80, 134115 (2009).

${ }^{11}$ J. D. Ju, D. Jang, A. Nwankpa, and M. Atzmon, J. Appl. Phys. 109, 053522 (2011).

${ }^{12}$ W. L. Johnson and K. Samwer, Phys. Rev. Lett. 95, 195501 (2005).

${ }^{13}$ R. Burridge and L. Knopoff, Bull. Seismol. Soc. Am. 57, 341 (1967).

${ }^{14}$ J. M. Carlson and J. S. Langer, Phys. Rev. A 40, 6470 (1989).

${ }^{15}$ J. M. Carlson and J. S. Langer, Phys. Rev. Lett. 62, 2632 (1989).

${ }^{16}$ B. A. Sun, H. B. Yu, W. Jiao, H. Y. Bai, D. Q. Zhao, and W. H. Wang, Phys. Rev. Lett. 105, 035501 (2010).

${ }^{17}$ B. A. Sun, S. Pauly, J. Tan, M. Stoica, W. H. Wang, U. Kühn, and J. Eckert, Acta. Mater. 60, 4160 (2012).

${ }^{18}$ J. Antonaglia, W. J. Wright, X. J. Gu, R. R. Byer, T. C. Hufnagel, M. LeBlanc, J. T. Uhl, and K. A. Dahmen, Phys. Rev. Lett. 112, 155501 (2014).

${ }^{19}$ Z. Han, W. F. Wu, Y. Li, Y. J. Wei, and H. J. Gao, Acta Mater. 57, 1367 (2009).

${ }^{20} \mathrm{~K}$. Binder and W. Kob, Glassy Materials and Disordered Solids: An Introduction to their Statistical Mechanics (World Scientific, 2005).

${ }^{21}$ S. Wiggins, Introduction to Applied Nonlinear Dynamical Systems and Chaos (Springer, 2003), p. 727.

${ }^{22}$ D. Bigoni and F. Dal Corso, Proc. R. Soc. London, Ser. A 464, 2365 (2008).

${ }^{23}$ V. A. Levashov, J. R. Morris, and T. Egami, Phys. Rev. Lett. 106, 115703 (2011). 
${ }^{24}$ Z. Y. Liu, G. Wang, K. C. Chan, J. L. Ren, Y. L. Huang, X. L. Bian, X. H. Xu, D. S. Zhang, Y. L. Gao, and Q. J. Zhai, J. Appl. Phys. 114, 033520 (2013).

${ }^{25}$ Z. Y. Liu, G. Wang, K. C. Chan, J. L. Ren, Y. L. Huang, X. L. Bian, X. H. Xu, D. S. Zhang, Y. L. Gao, and Q. J. Zhai, J. Appl. Phys. 114, 033521 (2013).
${ }^{26}$ M. Zink, K. Samwer, W. L. Johnson, and S. G. Mayr, Phys. Rev. B 73, 172203 (2006).

${ }^{27}$ W. Jiao, P. Wen, H. L. Peng, H. Y. Bai, B. A. Sun, and W. H. Wang, Appl. Phys. Lett. 102, 101903 (2013).

${ }^{28}$ W. H. Wang, Sci. Sin-Phys. Mech. Astron. 44, 396 (2014). 